УДК 621.373

\author{
А.А. Белоусов ${ }^{1,2}$, Ю.Н. Вольхин ${ }^{1}$, А.В. Гамиловская ${ }^{1,2}$, \\ А.А. Дубровская ${ }^{1}$, Е.В. Тихонов ${ }^{1,2}$ \\ ${ }^{1}$ ОАО «Центральное конструкторское бюро автоматики», Омск, Россия \\ ${ }^{2}$ Омский государственный технический университет, Омск, Россия \\ О ПРИМЕНЕНИИ МЕТОДОВ И СРЕДСТВ РАДИОФОТОНИКИ \\ ДЛЯ ОБРАБОТКИ СИГНАЛОВ ДЕЦИМЕТРОВОГО, \\ САНТИМЕТРОВОГО И МИЛЛИМЕТРОВОГО ДИАПАЗОНОВ \\ дЛИн волн
}

\begin{abstract}
Данная статья является вводной к серии статей по тематике радиофотоники, в которых будут описаны результаты обзорно-реферативных, теоретических и экспериментальных работ, проведенных сотрудниками и аспирантами ОАО «Центральное конструкторское бюро автоматики» и Омского государственного технического университета. Рассмотрены наиболее яркие и наиболее типичные примеры использования методов и средств радиофотоники для решения некоторых острых проблем техники СВЧ, которые практически не поддаются решению в рамках «традиционной» радиоэлектроники.

Ключевые слова: фотоника, радиофототника, микроволновая фотоника, радиофотонные аналоговые устройства, радиофотонные аналоговые тракты, радиофотонные аналоговые процессоры, радиофотонные автогенераторы гармонических сигналов, радиофотонные аналого-цифрровые преобразователи, радиофоотонные линии задержки, радиофротонные преобразователи частоты - смесители, радиофотонные амплитудные детекторы, радиофотонные умножители частоты, радиофотонные фазовые манипуляторы, электрооптический модулятор, модулятор Маха - Цандера, электропоглощающий модулятор, фротодетектор, оптоволоконный тракт, прямая модуляция, внешняя модуляция.
\end{abstract}

A.A. Belousov ${ }^{1,2}$, lu.N. Volkhin ${ }^{2}$, A.V. Gamilovskaia ${ }^{1,2}$, A.A. Dubrovskaia ${ }^{1}$, E.V. Tikhonov ${ }^{1,2}$

1JSC "Central Design Bureau of Automatics", Omsk, Russian Federation

${ }^{2}$ Omsk State Technical University, Omsk, Russian Federation

\title{
RADIOPHOTONICAL METHODS AND TOOLS USED FOR MICROWAVE ANALOG AND DIGITAL SIGNAL PROCESSING
}

This article is an introduction to a series of articles on the radiophotonics which will describe the results of an overview and abstracts, theoretical and experimental works that have been done staff and postgraduate students of "Central Design Bureau of Automatics" and Omsk State Technical University. This article will address the most striking and the most typical examples of the use of the methods and 
means of radiophotonics for solving some critical problems of microwave technology, which are almost impossible to solve within "traditional" electronics.

Keywords: photonics, radiophotonics, microwave photonics, radiophotonical analog devices, radiophotonical analog tracts, radiophotonic analog processor, radiophotonic active oscillator, radiophotonical analog to digital converters, radiophotonic delay line, radiophotonic mixer, radiophotonic amplitude detector, radiophotonic frequency multiplier, radiophotonical phase-shift modulator, electrooptical modulator, Mach-Zehnder modulator, electroabsorption modulator, photodetector, fiber-optic line, direct modulation, external modulation

\section{1. Введение}

В настоящий момент фотонные и, в частности, лазерные технологии широко применяются в промышленности и медицине, системах передачи и хранения информации, в изделиях военной и специальной техники (ВСТ), а также в быту и шоу-бизнесе. Информацию о таких приложениях фотоники можно найти не только в многочисленных научно-популярных и специализированных изданиях, но и в средствах массовой информации. Однако существуют и другие, менее известные, но не менее значимые для некоторых отраслей науки и техники фотонные технологии: например, фотонные технологии обработки высокочастотных радиосигналов дециметрового, сантиметрового и миллиметрового диапазонов длин волн (ДДВ). К сожалению, эти технологии неизвестны не только аудитории научно-популярных изданий, но и представителям даже тех отечественных профессиональных сообществ, которые имеют непосредственное отношение к разработке и исследованиям как в области фотоники, так и в области радиоэлектронных средств (РЭС) дециметрового, сантиметрового и миллиметрового ДДВ (ДМ-ДДВ, СМ-ДДВ и ММ-ДДВ соответственно). Отдельные попытки ученых $[1,2]$ привлечь внимание специалистов отечественной радиоэлектронной промышленности к этой перспективной тематике буквально до самого последнего времени каких-либо ощутимых результатов не давали. Поэтому основной целью данной вводной статьи будет ознакомление - хотя бы в общих чертах - читателей журнала «Прикладная фотоника» с некоторыми из известных авторам примерами использования фотонных технологий в трактах РЭС ДМ-ДДВ, СМ-ДДВ. Кроме того, в процессе изложения материала будет «волевыми» решениями вводиться некоторая терминология. Последнее обусловлено прежде всего тем, что нормативно утвержденная и даже общепринятая в профессиональном сообществе терминология по этой тематике отсутствует, а кроме того, эта терминология необходима для удобства изложения и восприятия излагаемого материала. 


\section{2. О причинах появления новой области науки и техники - радиофотоники}

Разработка перспективных РЭС ДМ-ДДВ, СМ-ДДВ и ММ-ДДВ для ВСТ - систем радиосвязи (РС), радионавигации (РН), радиоэлектронной борьбы (РЭБ), радиоэлектронной разведки (РЭР) и радиоэлектронного противодействия (РЭП), а также радиолокационных систем (РЛС) - практически всегда велась с использованием тех или иных элементов фотонной техники. При этом практически до самого конца XX в. эти элементы использовались только для создания различных устройств индикации и подсветки. Редкие попытки использования фотонных технологий для обработки высокочастотных сигналов, например применение акустооптических Фурье-преобразователей $[3,4]$, какого-либо существенного влияния на развитие РЭС для ВСТ не оказали. Последнее было обусловлено прежде всего тем, что такие устройства, по большому счету, так и не вышли за рамки «лабораторных» конструкций, которые плохо «сочетались» с теми конструктивными решениями, которые использовались - и до сих пор используются - при проектировании высокочастотных узлов и блоков РЭС для ВСТ. Кроме того, эти акустооптические устройства были и до сих пор остаются относительно низкочастотными. И даже такая авторитетная организация, как DARPA (Defense Advanced Research Projects Agency), судя по всему, не предпринимала каких-либо особо акцентированных усилий для того, чтобы фотоника «шагнула» в высокочастные тракты РЭС для ВСТ.

Однако положение дел кардинально изменилось после того, как были разработаны надежные и серийно воспроизводимые сверхширокополосные электрооптические модуляторы (ЭОМ), лазерные диоды с прямой модуляцией (ЛДПМ) и фотодетекторы (ФД), предназначенные для создания высокоскоростных волоконно-оптических линий передачи $\left(\right.$ ВОЛП $\left.{ }^{1}\right)$. При этом, в отличие от вышеупомянутых акустооптических Фурье-преобразователей, данные ЭОМ, ЛДПМ и ФД (рис. 1-3) были реализованы в виде герметизируемых гибридных интегральных схем (ГИС) с использованием практически тех же самых конструктивных и технологических решений, что и традиционные ГИС ДМ-ДДВ, СМ-ДДВ и ММ-ДДВ. В настоящий момент верхняя граница диапазона рабочих частот (ДРЧ) по электрическим входам/выходам у мелкосерий-

\footnotetext{
${ }^{1}$ ГОСТ 26599-85. Системы передачи волоконно-оптические. Термины и определения.
} 


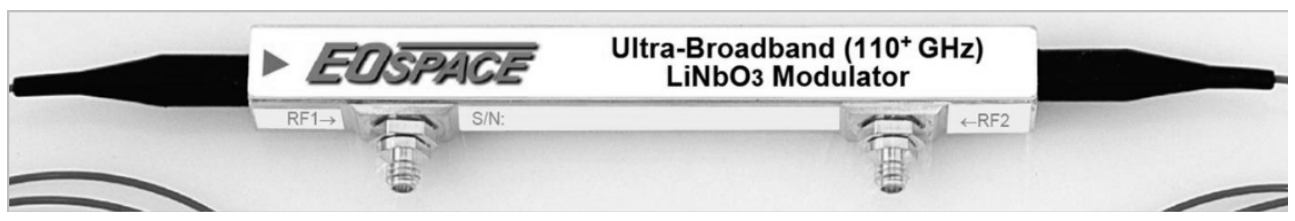

Рис. 1. ГИС сверхширокополосного ЭОМ

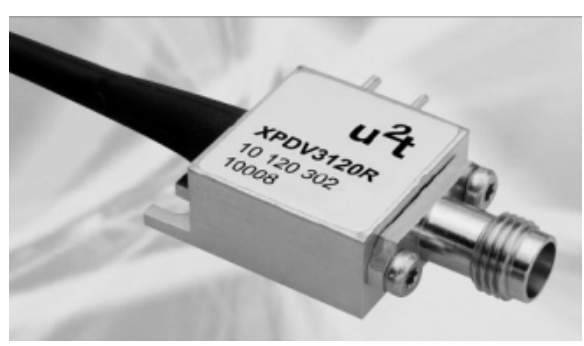

Рис. 2. ГИС сверхширокополосного ФД

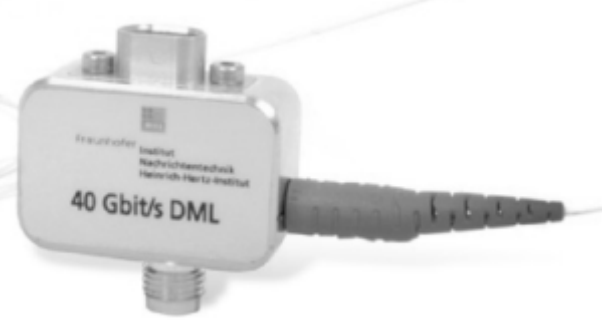

Рис. 3. ГИС ЛДПМ

ных коммерческих ГИС ЭОМ достигает 100-110 ГГц ${ }^{2}$, у ГИС ФД75 ГГц и выше ${ }^{3}$, а у ЛДПМ ${ }^{4}-30$ ГГц и выше.

Для разработки таких ГИС потребовались универсальные специалисты, которые должны были обладать профессиональной подготовкой в области фотоники, полупроводниковой техники, техники СВЧ и еще ряда научных и технических дисциплин. Или, другими словами, потребовались специалисты в новой, «гибридной» области науки и техники, в рамках которой исследуются взаимодействия оптических и высокочастотных электрических полей (сигналов, волн), а также создаются технические устройства, принцип работы которых основан на таких взаимодействиях. В нашей стране эта область науки и техники какого-либо нормативно утвержденного наименования до сих пор не имеет, а в профессиональном сообществе она иногда называлась радиооптикой $[2,5]$, иногда - СВЧ-оптикой [6], иногда сверхвысокочастотной оптоэлектроникой [1]. Однако в последнее время все чаще используется термин «радиофотоника», который, в частности, фигурировал в выступлении Председателя Правительства РФ Д.М. Медведева на заседании президиума Совета при Президенте РФ по модернизации

\footnotetext{
${ }^{2}$ www.gigoptix.com, www.eospace.com.

${ }^{3}$ www.u2t.com.

${ }^{4}$ www.hhi.fraunhofer.de.
} 
экономики и инновационному развитию России, которое состоялось 9 июля 2014 г. в Екатеринбурге, а также в принятых по итогам этого заседания поручениях Правительства РФ ${ }^{5}$. В зарубежной англоязычной литературе - в подавляющем большинстве случаев - используется термин Microwave Photonics (микроволновая фотоника) и аббревиатура $\mathrm{MWP}^{6}$.

Введем еще несколько терминов. Упомянутые выше ГИС ЭОМ, ЛДПМ и ФД содержат в своем составе как «электрические» тракты, по которым распространяются электромагнитные волны ДМ-ДДВ, СМ-ДДВ и ММ-ДДВ, так и оптические - фотонные - тракты, по которым распространяются электромагнитные волны оптического, а точнее, инфракрасного ДДВ. Поэтому такую элементную базу, в отличие от электронной компонентной базы (ЭКБ), будем в дальнейшем называть радиофотонной компонентной базой (РКБ). Также для удобства изложения и восприятия приведенного ниже текста введем еще один термин - фотонная компонентная база (ФКБ). Так предлагается называть те компоненты, которые содержат только оптические - фотонные - тракты.

С появлением вышеупомянутой РКБ, а также широкой номенклатуры активной и пассивной ФКБ возникла возможность реализации целой серии радиофотонных аналоговых устройств.

В целом все эти аналоговые радиофотонные устройства можно разделить на четыре группы: радиофотонные аналоговые тракты, радиофотонные аналоговые процессоры, радиофотонные автогенераторы гармонических сигналов, радиофотонные аналого-цифровые преобразователи.

\section{3. Радиофотонные аналоговые тракты}

В настоящий момент основным объектом приложения методов и средств радифотоники являются радиофотонные аналоговые тракты (РФАТ). Типичный РФАТ содержит (рис. 4) модуль электрооптического преобразования (МЭП), оптоволоконный тракт (ОВТ) и модуль оптоэлектронного преобразования (МОП).

В МЭП может осуществляться либо прямая модуляция оптического сигнала электрическим сигналом $U_{\text {вх }}(t)$, либо внешняя модуляция. В случае реализации прямой модуляции МЭП состоит из одного

\footnotetext{
${ }^{5}$ Решения по итогам заседания президиума совета по модернизации экономики и инновационному развитию [Электронный ресурс]. - URL: www.government.ru/orders/13808.

${ }^{6}$ www.mwp2014.com.
} 


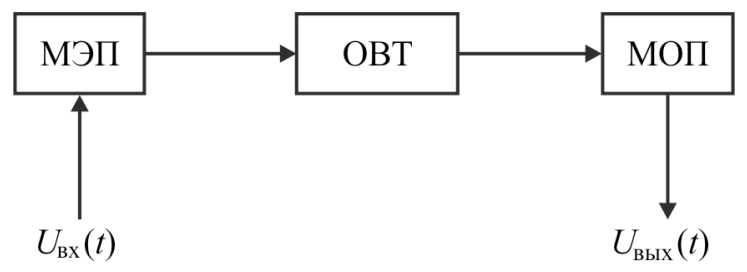

Рис. 4. Схема структурная РФАТ: МОП - модуль оптоэлектронного преобразования, МЭП - модуль электрооптического преобразования, ОВТ оптоволоконный тракт

ЛДПМ (рис. 5, a). В случае реализации внешней модуляции (рис. 5, б) в состав МЭП входит источник оптического излучения (ИОС) и электрооптический модулятор (ЭОМ). Как правило, в качестве ИОС используются лазерные диоды (ЛД), а в качестве ЭОМ - либо электропоглощающие модуляторы (ЭПМ), либо «интерференционные» ЭОМ. Принцип действия ЭПМ основан на эффекте Франца - Келдыша [7]. «Интерференционные» ЭОМ, как правило, реализованы по принципу интерферометра Маха-Цандера, а воздействие электрического сигнала на оптический осуществляется с использованием эффекта Поккельса [8]. Такие ЭОМ называют модуляторами Маха - Цандера (ММЦ). В качестве МОП, как правило, используются ФД (рис. 5, в), на электрическом выходе которых выделяется продетектированный сигнал $U_{\text {вых }}(t)$. В качестве ОВТ в подавляющем большинстве случаев может использоваться обычное «коммерческое» одномодовое волокно типа SMF-28 ${ }^{7}$.

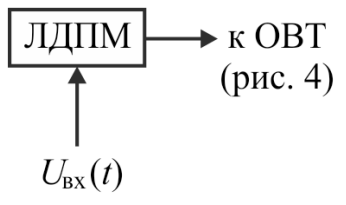

$a$

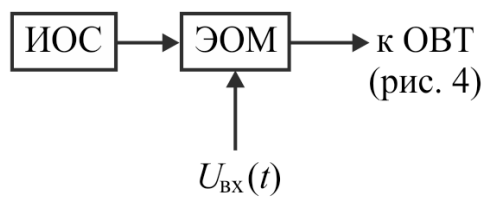

6

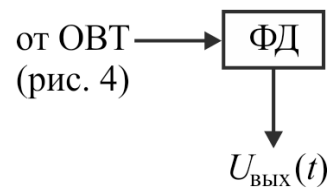

B

Рис. 5. Схемы структурные МЭП и МОП (см. рис. 4): $a-$ МЭП с прямой модуляцией; $\sigma$ - МЭП с внешней модуляцией; в - МОП: ИОС - источник оптического сигнала, ЛДПМ - лазерный диод с прямой модуляцией, ФД - фотодетектор, ЭОМ - электрооптический модулятор

\footnotetext{
${ }^{7}$ www.corning.com.
} 
В последующих статьях авторы рассмотрят особенности, достоинства и недостатки прямой и внешней модуляции, а также ЭПМ, ММЦ и других видов ЭОМ. А сейчас мы можем констатировать следующее: оптимальным вариантом при реализации широкополосных и сверхширокополосных РФАТ является использование МЭП с внешним видом модуляции и ЭОМ в виде ММЦ. Последнее обусловлено тем, что такая реализации РФАТ позволяет получить максимальную широкополосность: от постоянного тока до 70 ГГц и выше ${ }^{8}$; минимальные коэффициенты шума: ниже 10 дБ [9]; максимальные коэффициенты передачи $\left(K_{\text {п }}\right.$ $=20 \lg \left(U_{\text {вых }}(t) / U_{\text {вх }}(t)\right)$ : до 0 дБ и выше $[9,10]-$ и это без использования каких-либо малошумящих высокочастотных усилителей на входах и выходах РФАТ, а также оптических усилителей между МЭП и МОП. Поэтому в большинстве случаев мы будем рассматривать РФАТ, которые реализованы так, как показано на рис. 6 .

Такие РФАТ могут использоваться вместо обычных высокочастотных коаксиальных кабелей (КК). Эффект от замены одного из самых лучших и самых высокочастотных на настоящий момент КК типа LL160 фирмы Harbour Industries ${ }^{9}$ длиной 30 м на РФАТ с такой же длиной ОВТ (см. рис. 6) проиллюстрирован на рис. 7.

МЭП

МОП

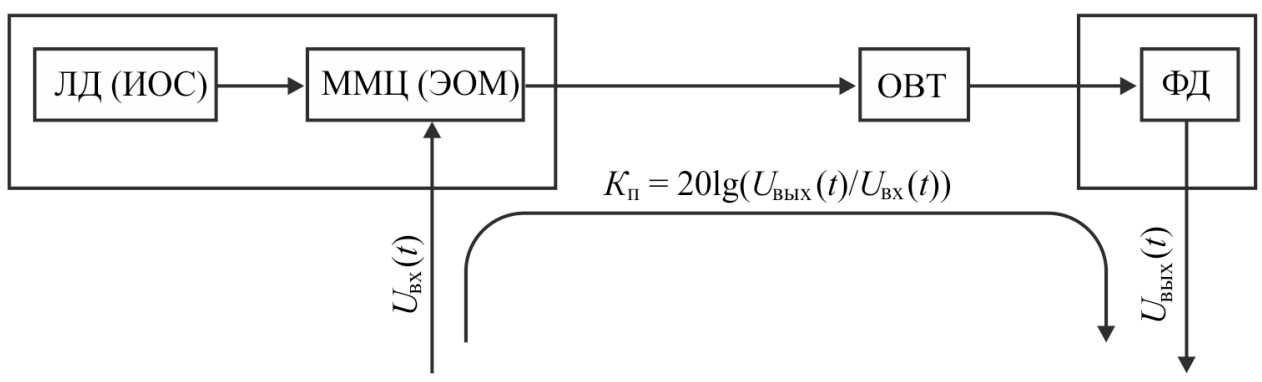

Рис. 6. Типичная схема структурная РФАТ (см. рис. 4) с внешней модуляцией: ИОС - источник оптического сигнала; ЛД - лазерный диод; ММЦ - модулятор

Маха - Цандера; МОП - модуль оптоэлектронного преобразования; МЭП модуль электрооптического преобразования; ОВТ - оптоволоконный тракт; ФД - фотодетектор; ЭОМ - электрооптический модулятор

\footnotetext{
${ }^{8}$ www.gigoptix.com, www.eospace.com, www.u2t.com.

${ }^{9}$ www.harbourind.com.
} 


\section{$F, \Gamma Г ц$}

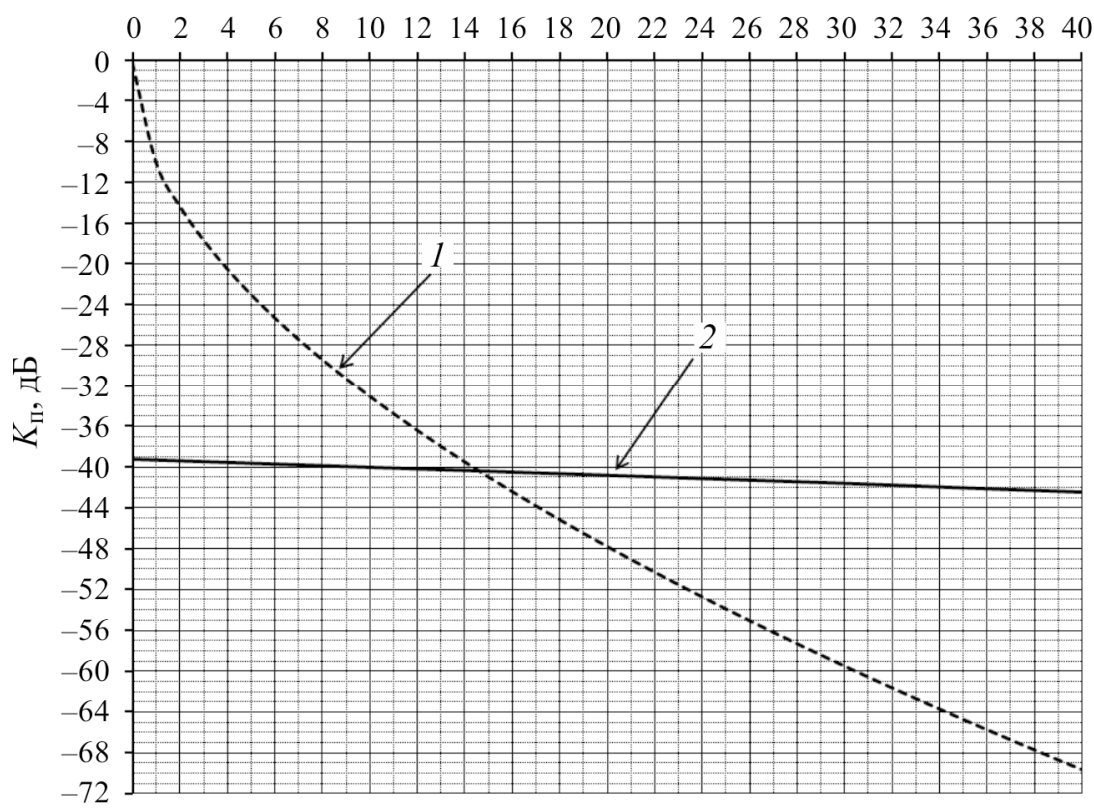

Рис. 7. Частотные зависимости $K_{\text {п }}$ высокочастотного коаксиального кабеля (кривая 1) и сверхширокополосного РФАТ (кривая 2)

Из рис. 7 видно, при переходе от КК к РФАТ мы увеличиваем

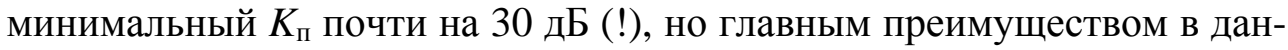
ном случае является не увеличение $K_{\text {п }}$, а уменьшение неравномерно-

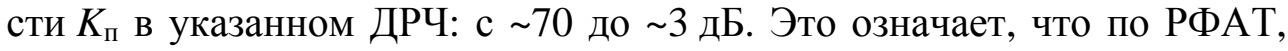
в отличие от КК, можно передавать сверхширокополосные сигналы с минимальными искажениями спектра, в частности, субнаносекундные радиоимпульсы от удаленных относительно простых и компактных антенных постов (АП), расположенных по периметру, например, «большого» самолета (рис. 8) к центральному посту (ЦП).

Этот ЦП можно расположить в относительно комфортных условиях внутри фюзеляжа, при этом не особо ограничивая габариты. Поэтому появляется возможность создать в ЦП мощнейшее устройство обработки принятых сигналов и тем самым возможность увеличить эффективность РЭС (РЭБ, РЭП, РЭР или РЛС), для реализации которой был использован вышеупомянутый сверхширокополосный РФАТ. Также с большой долей уверенности можно утверждать, что аналоговые РФАТ найдут широкое применение при создании перспективных образов различных фазированных антенных решеток [11-13]. 


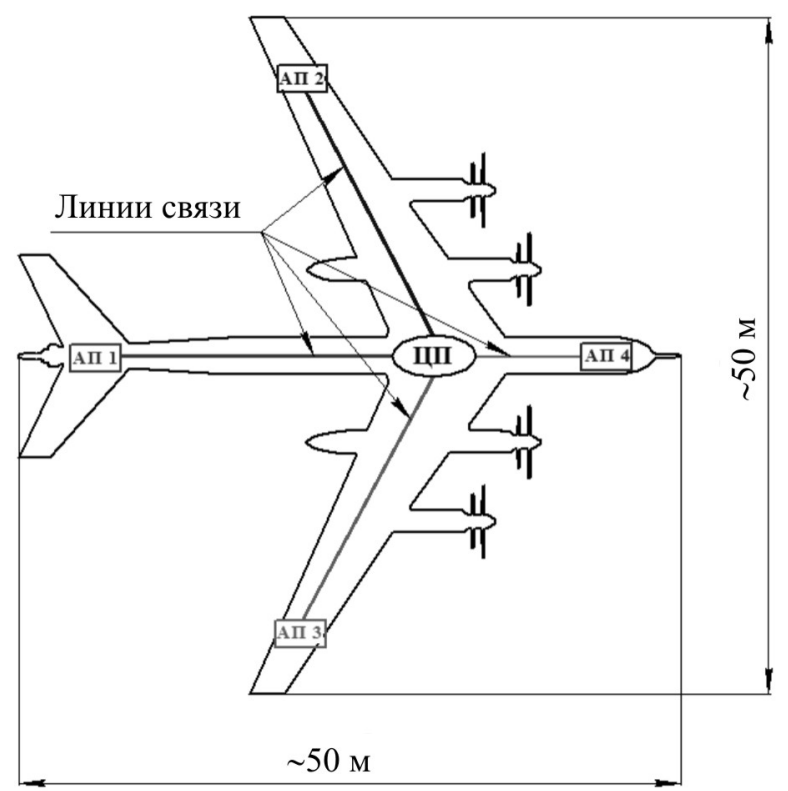

Рис. 8. Размещение АП и ЦП на «большом» самолете

Необходимо отметить, что приведенную на рис. 7 АЧХ будет иметь РФАТ, изготовленный с применением общедоступной «коммерческой» РКБ. В случае использования «специальной» РКБ коэффициент передачи РФАТ, как было сказано выше $[9,10]$, может быть увеличен на несколько порядков - вплоть до положительных значений.

Одними из лидеров на этом рынке РКБ является фирма Photonic Systems, которая в настоящий момент серийно производит, например, так называемые фотонные линки (photonic links) - пары МЭП и МОП (см. рис. 4) - серии PSI-2600 ${ }^{10}$ с ДРЧ до 20 ГГц (рис. 9), а в 2013 г. сотрудники этой фирмы сообщили о создании линка с ДРЧ до 100 ГГц [14].

Необходимо отметить, что рассказ об аналоговых РФАТ будет не полным, если не рассмотреть один из наиболее оригинальных вариантов реализации такого тракта, который был предложен в [15]. В данном случае мы имеем дело с внешней модуляцией (рис. 10), но осуществляется она и не в ЭПМ, и не в ММЦ, а в так называемой электрооптической антенне. Здесь, как и в ММЦ, воздействие электрического сигнала на оптический осуществляется с использованием эффекта Поккельса, но при этом модулирующий электрический сигнал, в отличие

${ }^{10}$ www.photonicsinc.com. 


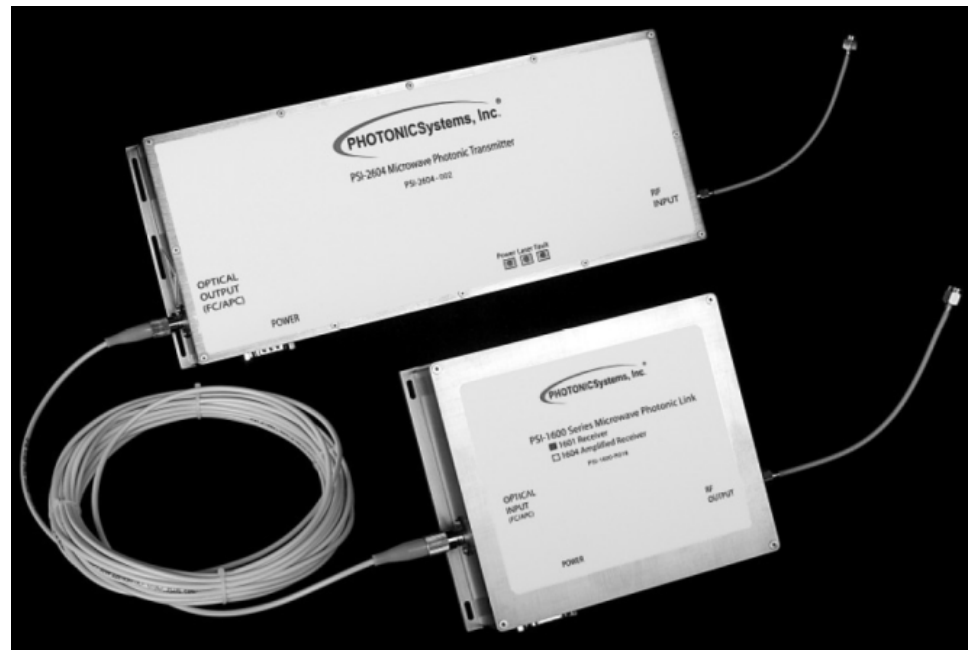

Рис. 9. Фотонные линки производства Photonic Systems

Электромагнитная волна

из эфира

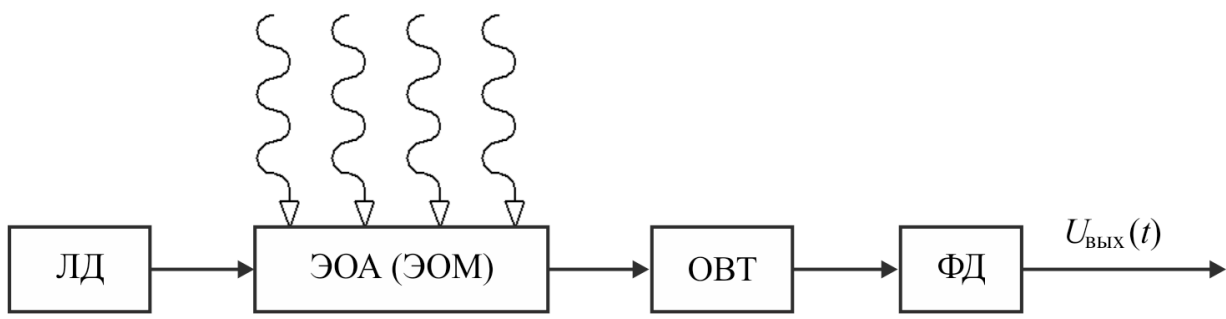

Рис. 10. РФАТ с ЭОМ в виде электрооптической антенны: ЛД - лазерный диод;

ОВТ - оптоволоконный тракт; ФД - фотодетектор; ЭОА - электрооптическая антенна; ЭОМ - электрооптический модулятор

от ММЦ, представляет собой «неканализированную» электромагнитную волну. Предложенное техническое решение позволяет создавать так называемые полностью неэлектронные входные каскады, в которых отсутствует гальваническая связь между металлическими элементами антенны (которые вообще могут отсутствовать) и входными клеммами полупроводниковых малошумящих усилителей, которые, как правило, устанавливаются на входах высокочувствительных РЭС для ВСТ. Поэтому такие РФАТ (см. рис. 10) могут выполнять функцию защитных устройств входных трактов РЭС от воздействия мощных электромагнитных излучений. В настоящий момент эти защитные устройства становятся крайне актуальными из-за появления так называе- 
мого электромагнитного или радиочастотного оружия [16], действующие образцы которого были разработаны, например, в рамках Counterelectronics High-powered Microwave Advanced Missile Project (CAMP), проводимого корпорацией Boeing по заказу Air Force Research Laboratory $(\mathrm{AFRL})^{11}$. К сожалению, пока такие защитные устройства, вероятнее всего, являются относительно узкополосными.

Очевидно, что потенциальный рынок РФАТ, в том числе широкополосных и сверхширокополосных, огромен, даже несмотря на то, что применяться эти РФАТ будут в подавляющем большинстве случаев для создания ВСТ. Поэтому можно утверждать, что и в настоящий момент, и в ближайшем будущем основным объектом приложения методов и средств радифотоники будут именно сверхширокополосные РФАТ ДМ-ДДВ, СМ-ДДВ и ММ-ДДВ.

\section{4. Радиофотонные аналоговые процессоры}

Функции различных радиофотонных аналоговых процессоров (РАП) могут выполнять РФАТ. При определенном подборе РКБ и ФКБ, а также энергетических режимов их эксплуатации РФАТ могут выполнять функции сверхширокополосных усилителей $[9,10]$, умножителей частоты на 2 [10], фазовых манипуляторов 0-180 [17] и высокочувствительных амплитудных детекторов [17], ДРЧ которых полностью перекрывает ДМ-ДДВ, СМ-ДДВ и часть ММ-ДДВ.

Изменяя длину ОВТ (см. рис. 4, 6), можно изменять время прохождения модулированного оптического сигнала между МЭП и МОП и, как следствие, время прохождения высокочастотного электрического сигнала от входа МЭП до выхода МОП. Поэтому РФАТ может выполнять функцию сверхширокополосной радиофотонной линии задержки, время задержки $(\tau)$ в которой будет довольно слабо зависеть от частоты электрического сигнала в широком диапазоне частот. При этом величина $\tau$ может достигать нескольких десятков микросекунд ${ }^{12}$.

В [18] предложено между МЭП и МОП разместить еще один ММЦ так, как показано на рис. 11. Такой РФАТ может выполнять функцию сверхширокополосного радиофотонного преобразователя частоты - смесителя. Рассмотрим принцип работы такого смесителя.

\footnotetext{
${ }^{11}$ www.fbo.gov.

${ }^{12}$ www.centervospi.ru.
} 


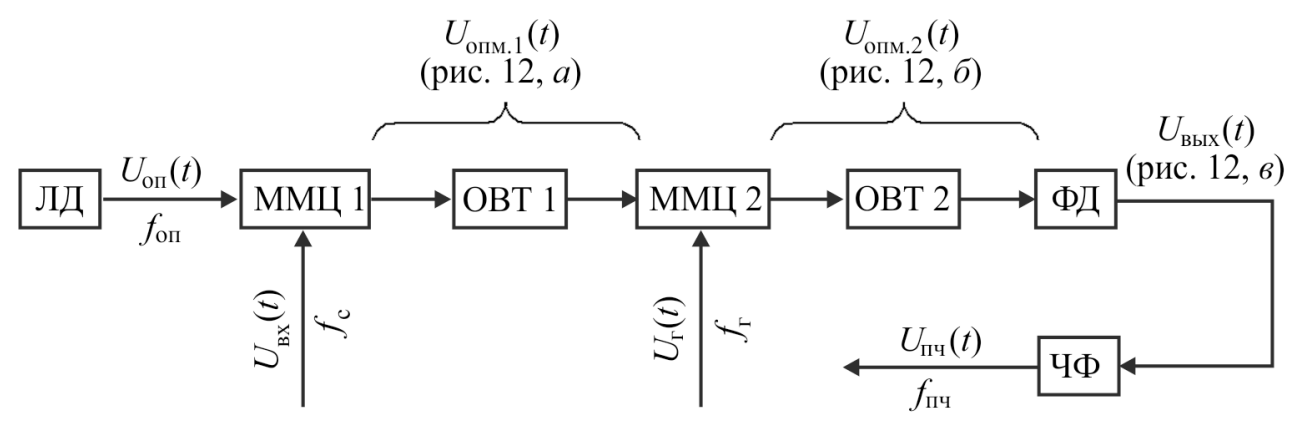

Рис. 11. Схема структурная сверхширокополосного радиофотонного смесителя: ЛД - лазерный диод; ММЦ 1, ММЦ 2 - модуляторы Маха - Цандера;

OВТ 1, ОВТ 2 - оптоволоконные тракты; ФД - фотодетектор; ЧФ - частотный фильтр

Сначала входным электрическим сигналом $U_{\text {вх }}(t)$ с частотой $f_{\mathrm{c}}$ в ММЦ 1 осуществляется амплитудная модуляция оптического сигнала от ЛД $U_{\text {оп }}(t)$ с частотой $f_{\text {оп }}$. Продуктом этой модуляции является амплитудно-модулированный оптический сигнал $U_{\text {опм.1 }}(t)$, в спектре которого присутствуют три основные спектральные составляющие (рис. 12, $a$ ): $\left(f_{\text {оп }}\right),\left(f_{\text {оп }}+f_{\mathrm{c}}\right)$ и $\left(f_{\text {оп }}-f_{\mathrm{c}}\right)$. Далее (см. рис. 11$)$ этот оптический сигнал с выхода ММЦ 1 через ОВТ 1 поступает на оптический вход ММЦ 2, на СВЧвход которого поступает сигнал гетеродина $U_{\text {г }}(t)$ с частотой $f_{\Gamma}$. В ММЦ 2 осуществляется вторичная модуляция оптического сигнала, и продуктом этой модуляции является также модулированный оптический сигнал $U_{\text {опм.2 }}(t)$, спектр которого содержит уже девять основных спектральных составляющих (рис. $12, \sigma):\left(f_{\text {оп }}\right),\left(f_{\text {оп }}+f_{\mathrm{c}}\right),\left(f_{\text {оп }}-f_{\mathrm{c}}\right),\left(f_{\text {оп }}+f_{\text {г }}\right),\left(f_{\text {оп }}-f_{\text {г }}\right)$, $\left(f_{\text {оп }}+f_{\mathrm{c}}-f_{\text {г }}\right),\left(f_{\text {оп }}+f_{\mathrm{c}}+f_{\text {г }}\right),\left(f_{\text {оп }}-f_{\mathrm{c}}-f_{\text {г }}\right)$ и $\left(f_{\text {оп }}-f_{\mathrm{c}}+f_{\text {г }}\right)$. Данный оптический сигнал с выхода ММЦ 2 через ОВТ 2 поступает на оптический вход ФД, на электрическом выходе которого формируется электрический выходной сигнал $U_{\text {вых }}(t)$. Спектр последнего содержит четыре основные спектральные составляющие (рис. 12,6$):\left(f_{\mathrm{c}}\right),\left(f_{\mathrm{r}}\right),\left(f_{\mathrm{c}}+f_{\mathrm{r}}\right),\left(f_{\mathrm{c}}-f_{\mathrm{r}}\right)$. Требуемая спектральная составляющая - выходной сигнал $U_{\text {пч }}(t)$ с частотой $f_{\text {пч }}-$ может быть выделена соответствующим частотным фильтром (ЧФ, см. рис. 11).

Можно предположить, что такие радиофотонные смесители могут иметь ДРЧ по входам $U_{\mathrm{c}}(t)$ и $U_{\mathrm{r}}(t)$ до 100-110 ГГц, а по выходу $U_{\text {вых }}(t)$ - до 70 ГГц и выше.

Однако наиболее типичная схема структурная РАП будет выглядеть так, как показано на рис. 13. В данном случае между МЭП и МОП включен модуль аналоговой фотонной обработки (МАФО). 


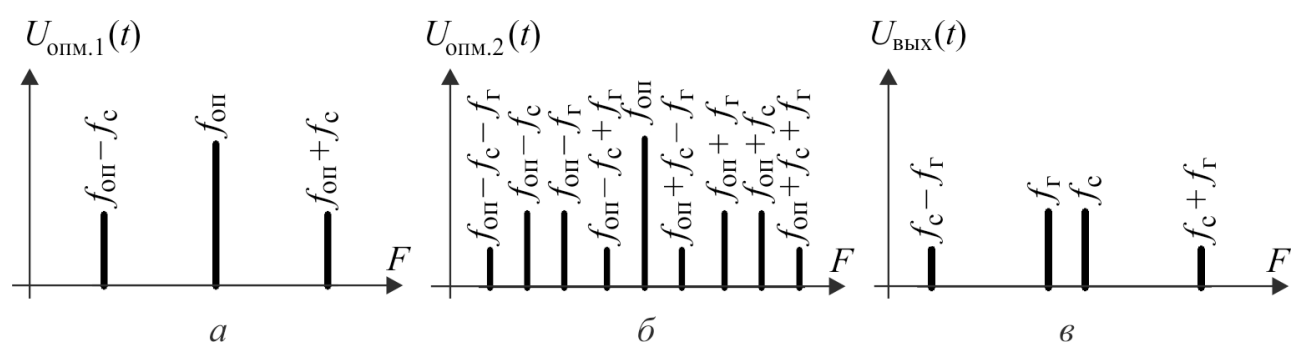

Рис. 12. Спектры сигналов в различных точках тракта сверхширокополосного радиофотонного смесителя (см. рис. 11)

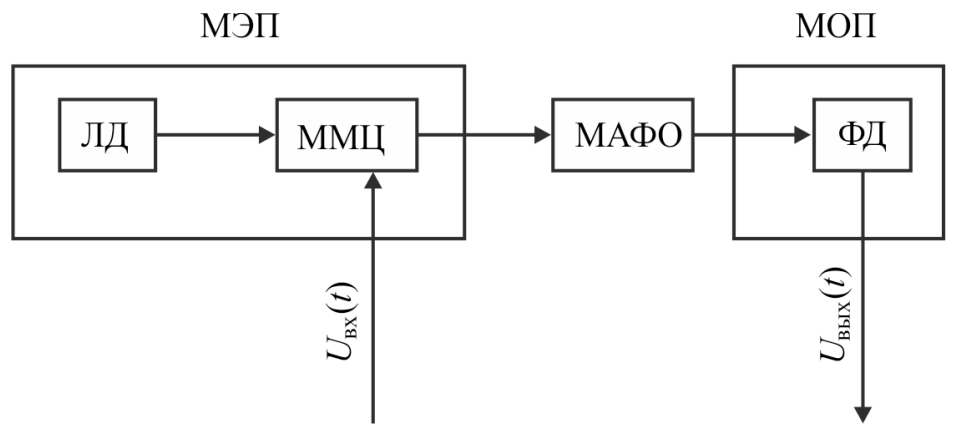

Рис. 13. Типичная схема структурная РАП: ЛД - лазерный диод; МАФО - модуль аналоговой фотонной обработки; ММЦ - модулятор Маха-Цандера; ФД - фотодетектор

В качестве МАФО может использоваться самая различная ФКБ. Так, например, в [18] описан радиофотонный приемник, схема структурная которого приведена на рис. 14.

На выходе данного приемника при помощи 16-канального оптического разветвителя, 16 оптических фильтров Фабри - Перо и 16 фотодетекторов из входного потока сигналов СВЧ $\left(U_{\mathrm{Bx}}(t)\right)$ формируется 16 выходных квази-НЧ сигналов $\left(U_{\text {вых1 }}(t)-U_{\text {вых16 }}(t)\right)$, амплитуда которых прямо пропорциональна уровню мощности спектральных составляющих в том или ином фрагменте спектра $U_{\text {вх }}(t)$. Фактически данный приемник выполняет функцию относительно грубого радиофотонного Фурье-преобразователя с разрешающей способностью 1 ГГц в полосе частот 16 ГГц. Необходимо отметить, что в настоящий момент существуют такие оптические фильтры, которые позволяют обеспечить разрешающую способность до 200 МГц и менее [19].

В настоящий момент существующая ФКБ, в том числе серийно выпускаемая, позволяет создавать широкую номенклатуру архитектур МАФО, предназначенных для аналоговой обработки модулированных 
в МЭП оптических сигналов и в частотной, и во временной области. Возможные варианты реализации таких МАФО будут рассматриваться в последующих статьях.

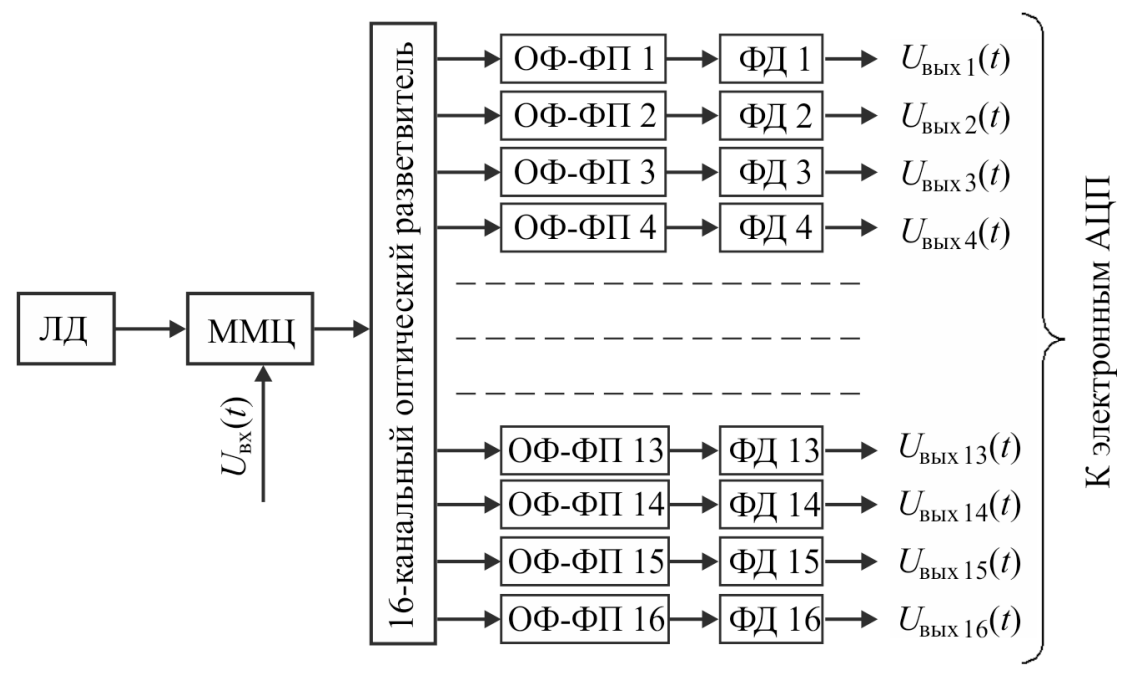

Рис. 14. Структурная схема радиофотонного многоканального приемника: ЛД - лазерный диод; ММЦ - модулятор Маха - Цандера;

ОФ-ФП 1 - ОФ-ФП 16 - оптические фильтры Фабри - Перо; ФД 1 - ФД 16 - фотодетекторы

В заключение разговора об РАП необходимо отметить следующее. В настоящий момент на рынке предлагаются пакеты систем автоматизированного проектирования (САПР), предназначенных для проектирования различных радиофотонных устройств, в том числе РАП, например пакет САПР фирмы Optiwave Systems ${ }^{13}$. Появление таких САПР на рынке, а также перечень пользователей этих САПР - от General Electric и Boeing до Alcatel-Lucent и Hitachi, от Lockheed Martin и Photonic Systems до Sony и Intel - говорит об актуальности разработки радиофотонных аналоговых устройств вообще и РАП в частности.

\section{5. Радиофотонные автогенераторы гармонических сигналов}

В некоторых источниках [20] описаны так называемые оптоэлектронные генераторы (ОЭГ) или, иначе, радиофотонные автогенераторы

\footnotetext{
${ }^{13}$ www.optiwave.com.
} 
гармонических сигналов (РАГС). На рис. 15 приведена упрощенная схема такого РАГС.

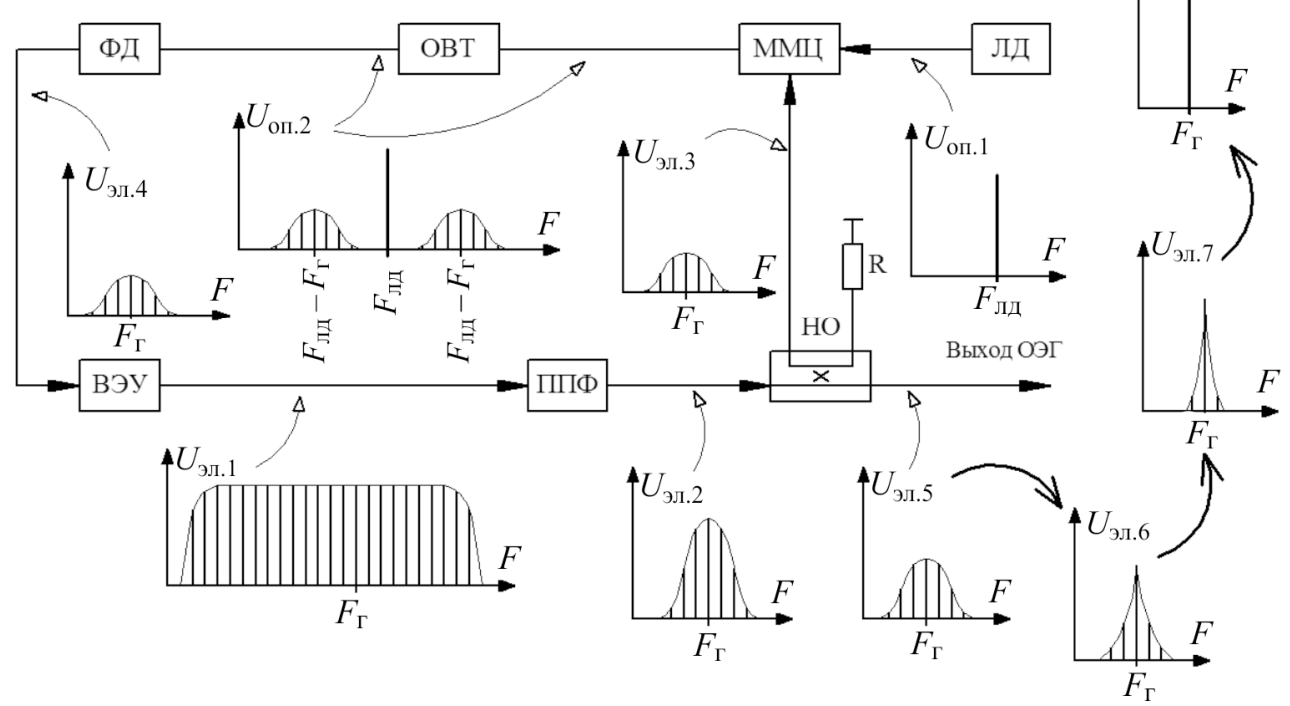

Рис. 15. Структурная схема простейшего РАГС: ВЭУ - высокочастотный электронный усилитель; ЛД - лазерный диод; ММЦ - модулятор Маха - Цандера;

$\mathrm{HO}$ - направленный ответвитель; ОВТ - оптоволоконный тракт; ППФ - полоснопропускающий фильтр; ФД - фотодетектор

Принцип работы РАГС можно описать следующим образом. Широкополосный шумовой сигнал $\left(U_{\text {эл.1 }}\right)$ с выхода высокочастотного электронного усилителя (ВЭУ) поступает на вход полосно-пропускающего фильтра (ППФ). Этот ППФ формирует узкополосный шумовой сигнал $\left(U_{\text {эл.2 }}\right)$. Часть этого шумового сигнала $\left(U_{\text {эл.3 }}\right)$ ответвляется направленным ответвителем (НО) и поступает на электрический вход ММЦ. На оптический вход ММЦ поступает сигнал от ЛД $\left(U_{\text {оп.1 }}\right)$. На

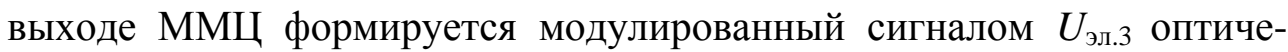
ский сигнал $\left(U_{\text {оп.2}}\right)$, который через отрезок ОВТ поступает на вход ФД. На выходе фотодетектора формируется электрический сигнал $U_{\text {эл.4 }}$, который поступает на вход ВЭУ. Таким образом формируется цепь положительной обратной связи и создаются условия для самовозбуждения автогенератора. В роли «резонансного» элемента этой обратной связи выступает отрезок ОВТ. Если напряжение питания на ВЭУ подается после того, как поданы напряжения питания на ЛД и ММЦ, то в первый момент времени ширина спектра сигнала на выходе прямого 


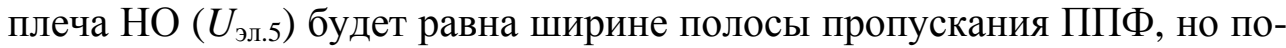
сле каждого цикла прохождения электрических и оптических сигналов по трактам между выходом ВЭУ и его входом ширина спектра на выходе прямого плеча $\mathrm{HO}$ - и собственно на выходе РАГС - будет все уже и уже, а амплитуда спектральных составляющих в окрестностях

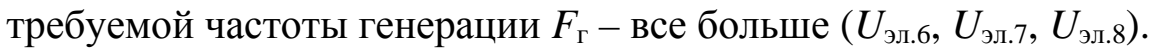

Используя такое схемотехническое решение, можно получать гармонические сигналы, параметры спектра которых в ДД-ДДВ, СМ-ДДВ и даже ММ-ДДВ могут быть соизмеримы с параметрами низкочастотных автогенераторов метрового ДДВ, частоты которых стабилизируются кварцевыми резонаторами. А если в цепи обратной связи использовать более добротные, чем отрезки ОВТ, фотонные элементы, то параметры спектра выходных сигналов могут быть улучшены на порядок.

Необходимо отметить, что, используя приведенное на рис. 15 схемотехническое решение, можно создавать не только РАГС с фиксированной $F_{\text {г }}$, но и перестраиваемые РАГС. Перестройка частоты в таких РАГС может осуществляться, например, при помощи перестраиваемого ППФ, а также с использованием некоторых других способов [21].

Одним из лидеров на рынке таких высокостабильных РАГС ДМ-ДДВ, СМ-ДДВ и ММ-ДДВ является фирма OEwaves ${ }^{14}$.

Результаты экспериментальных и теоретических работ по данной тематике авторы планируют представить в последующих статьях.

\section{6. Радиофотонные аналого-цифровые преобразователи}

С высокой долей уверенности можно утверждать, что наибольший эффект применения методов и средств радиофотоники в трактах РЭС для ВСТ может быть достигнут в том случае, если будут созданы сверхширокополосные радиофотонные аналого-цифровые преобразователи (РАЦП) с ДРЧ, которые перекрывают часть ДМ-ДДВ, весь СМ-ДДВ и часть ММ-ДДВ. При этом речь идет о таких АЦП, в которых дискретизация входного электрического сигнала осуществляется в соответствии с требованиями теоремы Котельникова [22]. На уровне физического эксперимента это уже возможно [23]. Также авторам известно о попытках создания полупроводниковых РКБ и ЭКБ, а также ФКБ [24], необходимых для создания дееспособных промышленных

\footnotetext{
${ }^{14}$ www.oewaves.com.
} 
образцов РАЦП. К сожалению, данная тематика является для отечественных ученых и инженеров абсолютно новой, а решение проблем, которые могут возникнуть при создании РАЦП, возможно только на таком уровне развития науки техники, на который отечественным специалистам еще только предстоит выйти. Поэтому в данный момент мы можем только обозначить в самых общих чертах перечень возможных схемотехнических решений, а также перечень тех проблем, которые придется решить в самых различных областях науки и техники. В рамках одной статьи это сделать невозможно, и потому авторы планируют посвятить тематике РАЦП серию обзорных статей.

\section{7. Заключение}

Приведенные выше примеры реализации различных радиофотонных устройств наглядно показывают, что радиофотоника в большей степени является разделом радиоэлектроники ДМ-ДДВ, СМ-ДДВ и ММ-ДДВ или, как принято это назвать в отечественном профессиональном сообществе, техники СВЧ (что является абсолютно «ненормативным» термином ${ }^{15}$ ). Однако для решения проблем техники СВЧ методами и средствами радиофотоники необходимо создать высокотехнологичную и наукоемкую РКБ, а также весьма «эксклюзивную» ФКБ. Bce это потребует проведения широкого спектра научноисследовательских работ в самых различных областях науки и техники. Для проведения таких работ придется привлекать значительные финансовые и, что самое главное, высококвалифицированные кадровые ресурсы. И если с первым в последнее время дела у нас обстоят все лучше и лучше, то со вторым - все хуже и хуже. И потому мы не можем себе позволить нерачительное использование наших весьма скудных кадровых ресурсов. В то же время одной из важнейших задач на данном этапе развития радиофотоники в нашей стране является даже не решение каких-то конкретных научных и технических проблем, а формирование перечня тех проблем, которые являются актуальными для развития радиофотоники. Авторы статьи надеются, что сотрудничество с журналом «Прикладная фотоника» будет способствовать решению данной задачи.

\footnotetext{
${ }^{15}$ ГОСТ 24375-80. Радиосвязь. Термины и определения.
} 


\section{Список литературы}

1. Белкин М.Е., Сигов А.С. Новое направление фотоники - сверхвысокочастотная оптоэлектроника // Радиотехника и электроника. 2009. - Т. 54, № 8. - С. 901-914.

2. Червяков Г.Г., Роздобудько В.В. Введение в радиооптику. - М.: Учебная литература, 2009. - 260 с.

3. Акустооптические анализаторы спектра радиосигналов / А.П. Белошицкий, В.М. Комаров, Б.П. Крекотень, Б.Т. Сапожников // Зарубежная радиоэлектроника. - 1979. - № 3. - С. 51-70.

4. Патент РФ № 213019. Акустооптический измеритель параметров радиосигналов / В.В. Роздобудько, Г.С. Крутчинский; опубл. 10.05.1999.

5. Зверев В.А. Радиооптика. Преобразование сигналов в радио и оптике. - М.: Сов. радио, 1975.

6. Корнблит С. СВЧ оптика. Оптические принципы в приложении к конструированию СВЧ антенн: пер. с англ. / под. ред. О.П. Фролова. М.: Связь, 1980. - 360 с.

7. Исследование параметров модуляторов лазерного излучения на эффекте Франца-Келдыша / А.Н. Георгобиани [и др.] // Квантовая электроника. - 1980. - Т. 7, № 3. - С. 624-626.

8. Сивухин Д.В. Общий курс физики. - 3-е изд. - T. IV. Оптика. М.: Физматлит, 2002. - 792 с.

9. Signal-to-noise performance of two analog photonic links using different noise reduction techniques / E. Ackerman, G. Betts, W. Burns, J. Campbell, C. Cox, N. Duan, J. Prince, M. Regan, H. Roussell // IEEE MTT-S Int. Microwave Symp. Dig., Honolulu, Hawaii. - June 2007. - P. 51-54.

10. Вольхин Ю.Н., Гамиловская А.В. О возможности реализации сверхширокополосных аналоговых радиофотонных трактов диапазона СВЧ с положительными коэффициентами передачи // Материалы XVIII координационного научно-технического семинара по СВЧ-технике (Нижегородская область, п. Хахалы). - Хахалы, 2013.

11. Активные фазированные антенные решетки / под ред. Д.И. Воскресенского, А.И. Канащенкова. - М.: Радиотехника, 2004. - 488 с.

12. Optical techniquest to feed and control GaAs MMIC modules for phased array antenna applications / K.B. Bhasin, G. Anzic, R.R. Kunath, D.J. Connoly // Proc. AIAA $11^{\text {th }}$ Communication Satellite Systems Conf. New York, 1986. - P. 506-513. 
13. Lee J.J. RF Photonics for Beamforming and Array Applications // Paper presented at the RTO SET Lecture Series on "Optics Microwave Interactions". Held in Jouli en Josas. France, 2-3 September 2002; Duisburg Germany, 5-6 September 2002; Budapest, Hunganu, 9-10 September 2002, and published in RTO-EN-028.

14. Cox C.H., Ackerman E.I.. A Path to Realizing High-Performance 100-GHz Analog Links // IEEE Avionics, Fiber-Opticsand Photonics Conference (AVFOP). - 2013.

15. Patent № US 7450790 B1. Non-electronic radio frequency frontend with immunity to electromagnetic pulse damage / Bahram Jalali, Chia-Jen Hsu, Bijan Houshmand.

16. Прищепенко А. Электромагнитное оружие: аннибал у ворот // Мир Оружия. - 2005. - № 2.

17. Белоусов А.А., Вольхин Ю.Н., Дубровская А.А. Обзор и исследование возможных вариантов реализации сверхширокополосных детекторов, смесителей и других аналоговых процессоров диапазона СВЧ с использованием методов и средств радиофотоники // Обмен опытом в области создания сверхширокополосных радиоэлектронных систем: материалы науч.-техн. конф. - Омск: ЦКБА, 2014. - С. 37-61.

18. Manka M.E. Microwave Photonics for Electronic Warfare Applications // Microwave photonics, 2008. Jointly held with the 2008 asia-pasific microwave photonics conference. DOI: 10.1109/MWP.2008.4666690.

19. Chan E.H.W., Minasian R.A. Tuneable High-Q Microwave Photonic Bandpass Filter Without Coherent Interference Limitations // The $40^{\text {th }}$ European Microwave Conference (EuMC 2010). EuMC Poster18: New and Emerging Technologies and Materials. - 2010.

20. Opto-electronic Oscillator: Applications to Sensors / N.L. Duy, B. Journet, I. Ledoux-Rak, J. Zyss, L.V. Hai Nam, V.V. Luc // IEEE International Meeting on Microwave Photonics. - 2008.

21. Ultra-Broadband and Low Phase Noise Photonic Millimeter-Wave Generation / S. Fedderwitz, V. Rymanov, M. Wei $\beta$, A. Stöhr, D. Jäger, A.G. Steffan, A. Umbach // IEEE International Meeting on Microwave Photonics. -2008 .

22. Баскаков С.И. Радиотехнические цепи и сигналы: - М.: Высшая школа, 1983. $-536 \mathrm{c}$.

23. Optically Sampled Analog-to-Digital Converters / P.W. Juodawlkis, J.C. Twitchell, G.E. Betts, J.J. Hargreaves, R.D. Younger, J.L. Wasserman, 
F.J. O'donnell, K.G. Ray, R.C. Williamson // IEEE Transactions on Microwave Theory and Techniques. - 2001. - Vol. 49, № 10, part 2. - P. 1840-1853.

24. Patent 6,326,910 B1, USA. Photonic analog-to-digital conversion using light absorbers / Hayduk [et al.]. 2001.

\section{References}

1. Belkin M.E., Sigov A.S. Novoe napravlenie fotoniki - sverkhvysokochastotnaia optoelectronika [The new direction of photonics - the ultra-high frequency optoelectronics]. Radiotehnika i elektronika, 2009, vol. 54, no. 8, pp. 901-914.

2. Chervyakov G.G., Rozdobudko V.V. Vvedenie v radiooptiku [Introduction to radio optics]. Moscow: Uchebnaia literatura, 2009, $260 \mathrm{p}$.

3. Beloshitskii A.P., Komarov V.M., Krekoten' B.P., Sapozhnikov B.T. Akustoopticheskie analizatory spektra radiosignalov [Acoustooptical radio signal spectrum analyzers]. Zarubezhnaia radioelektronika, 1979, no. 3, pp. 51-70.

4. Rozdobudko V.V., Krutchinskii G.S. Acustoopticheskii izmeritel' parametrov radiosignalov [Acoustooptical radio signal meter]. RU Patent No. 2130192, published on 10.05.1999.

5. Zverev V.A. Radiooptika. Preobrazovanie signalov v radio i optike [Radio optics. Signal conversion in radio and optic areas]. Moscow: Sovetskoe radio, 1975.

6. Kornblit S. SVCh-optika. Opticheskie printsipy v prilozhenii k konstruirovaniiu SVCh-antenn [Microwave Optics. The Optics of Microwave Antenna Design]. Ed. O.P. Frolov. Moscow: Sviaz', 1980, 360 p.

7. Georgobiani A.N. [et. al.] Issledovanie parametrov moduliatorov lasernogo izlucheniia na effekte Franca-Keldisha [Parameter analysis of laser emission modulators on the basis of Frants-Keldysh effect]. Kvantovaia elektronika, 1980, vol. 7, no. 3, pp. 624-626.

8. Sivukhin D.V. Sivukhin D.V. Obshchii kurs fiziki. Tom IV. Optika [General course of physics. Vol. IV. Optics]. Moscow: Fizmatlit, 2002. - 792 p.

9. AckermanE., Betts G., Burns W., Campbell J., Cox C., Duan N., Prince J., Regan M., Roussell H. Signal-to-noise performance of two analog photonic links using different noise reduction techniques. IEEE MTT-S Int. Microwave Symp. Dig. Honolulu, Hawaii, 2007, pp. 51-54.

10. Vol'khin Iu.N., Gamilovskaia A.V. O vozmozhnosti realizatsii sverkhshirokopolosnykh analogovykh radiofotonnykh traktov diapazona SVCh 
s polozhitel'nymi koeffitsientami peredachi [Ability to implement ultrawideband microwave analog radiophotonic transmission lines with positive coefficients]. Materialy XVIII koordinatsionnogo nauchno-tekhnicheskogo seminara po SVCh-tekhnike. Nizhny Novgorod region, Hahali, 2013.

11. Voskresenskogo D.I., Kanashchenkova A.I. Aktivnye fazirovannye antennye reshetki [Active phased array antennas]. Moscow: Radiotehnika, 2004, 488 p.

12. Bhasin K.B., Anzic G., Kunath R.R., Connoly D.J. Optical techniquest to feed and control GaAs MMIC modules for phased array antenna applications. Proc. AIAA $11^{\text {th }}$ Communication Satellite Systems Conf., March 1986. New York, 1986, pp. 506-513.

13. Lee J.J. RF Photonics for Beamforming and Array Applications. Paper presented at the RTO SET Lecture Series on "Optics Microwave Interactions". Held in Jouli en Josas. France, 2-3 September 2002; Duisburg Germany, 5-6 September 2002; Budapest, Hunganu. 9-10 September 2002, and published in RTO-EN-028.

14. Cox C.H., Ackerman E.I. A Path to Realizing High-Performance 100-GHz Analog Links. IEEE Avionics, Fiber-Optics and Photonics Conference (AVFOP), 2013.

15. Bahram Jalali, Chia-Jen Hsu, Bijan Houshmand. Non-electronic radio frequency front- end with immunity to electromagnetic pulse damage. Patent № US 7450790 B1.

16. Prishchepenko A. Elektromagnitnoe oruzhie: annibal $\mathrm{u}$ vorot [Electromagnetic arms: Annibal at gate]. Mir Oruzhiia, 2005, no. 2.

17. Belousov A.A., Vol'khin Iu.N., Dubrovskaia A.A. Obzor i issledovanie vozmozhnykh variantov realizatsii sverkhshirokopolosnykh detektorov, smesitelei i drugikh analogovykh protsessorov diapazona SVCh s ispol'zovaniem metodov i sredstv radiofotoniki [Possible implementation on of ultrawideband microwave detectors, mixers and other analog processors using methods and means of radiophotonics]. Materialy nauchno-tekhnicheskoi konferentsii "Obmen opytom $v$ oblasti sozdaniia sverkhshirokopolosnykh radioelektronnykh system”. Omsk, 2014.

18. Manka M.E. Microwave Photonics for Electronic Warfare Applications. Microwave photonics, 2008. Jointly held with the 2008 asia-pasific microwave photonics conference. DOI: 10.1109/MWP.2008.4666690.

19. Chan E.H.W., Minasian R.A. Tuneable High-Q Microwave Photonic Bandpass Filter Without Coherent Interference Limitations. The $40^{\text {th }}$ 
European Microwave Conference (EuMC 2010). EuMC Poster18: New and Emerging Technologies and Materials, 2010.

20. Duy N.L., Journet B., Ledoux-Rak I., Zyss J., Hai Nam L.V., Luc V.V. Opto-electronic Oscillator: Applications to Sensors. IEEE International Meeting on Microwave Photonics, 2008.

21. Fedderwitz S., Rymanov V., Weiß M., Stöhr A., Jäger D., Steffan A.G., Umbach A. Ultra-Broadband and Low Phase Noise Photonic MillimeterWave Generation. IEEE International Meeting on Microwave Photonics, 2008.

22. Baskakov S.I. Radiotekhnicheskie tsepi i signaly [Radio engineering circuits and signals]. Moscow: Visshaia shkola, 1983, 536 p.

23. Juodawlkis P.W., Twitchell J.C., Betts G.E., Hargreaves J.J., Younger R.D., Wasserman J.L., O'donnell F.J., Ray K.G., Williamson R.C. Optically Sampled Analog-to-Digital Converters. IEEE Transactions on Microwave Theory and Techniques, 2001, vol. 49, no. 10, part 2, pp. 1840-1853.

24. Hayduk [et al.]. Photonic analog-to-digital conversion using light absorbers. Patent 6,326,910 B1, USA. 2001.

Получено 17.10.2014 